\title{
Infrared variability of BL Lacertae
}

\author{
J.H. Fan ${ }^{1,2}$, G.Z. Xie ${ }^{3}$, R.G. Lin $^{2}$, and Y.P. Qin ${ }^{3}$ \\ 1 CCAST (World Laboratory), P.O. Box 8730, Beijing 100080, China \\ 2 Center for Astrophysics, Guangzhou Normal University, Guangzhou 510400, China \\ e-mail: jhfan@guangztc.edu.cn \\ 3 Yunnan Observatory, Chinese Academy of Sciences, Kunming 650011, China
}

Received November 24, 1997; accepted June 10, 1998

\begin{abstract}
In this paper, the historic light curves of BL Lacertae in the infrared $(J H K)$ bands have been constructed from the published literature. Maximum variations of $\Delta J=2.29, \Delta H=2.42$, and $\Delta K=2.93$ and average color indices of $J-H=0.78 \pm 0.11, H-K=$ $0.78 \pm 0.12$, and $J-K=1.55 \pm 0.13$ have been found. The relation between the color index and the brightness of the source and the correlation of color-color indices have been investigated. Our analysis shows that there is no clear correlation between the color index and the brightness, and the emission mechanism is non-thermal in the nearinfrared.
\end{abstract}

Key words: infrared: galaxies — BL Lacertae objects: BL Lac

\section{Introduction}

BL Lacertae objects are extremely active galactic nuclei (AGNs) showing rapid and large variations, high and variable polarization, having no or weak $(E W<5 \AA)$ emission lines. In the last catalogue of active galaxies VeronCetty \& Veron (1996) compiled 220 BL Lac objects. BL Lac objects are violently variable not only in the optical (see Takalo et al. 1996 for the monitoring group: Florid group (Pica et al. 1980), Hamburg group (Schramm et al. 1994), Chinese group (Xie et al. 1994), Tuorla observation (Sillanpaa et al. 1991; Takalo et al. 1992) but also in the radio, infrared, UV, X-ray, and $\gamma$-ray bands (Tornikoski et al. 1994; Bregman et al. 1990; Soifer \& Neugebauer 1980; Impey et al. 1984; Holmes et al. 1984; Urry 1984; Thompson et al. 1995, 1996). It has been shown that the spectral indices of some objects change with the brightness of the source; generally the spectrum becomes flat while the source brightens (Morini et al. 1986; George et al. 1988; Fan 1993). In contrast, the BL Lac object 3C 66A shows a different behaviour (De Diego et al. 1997).
Polarization, which is a characteristic of BL Lac objects, is variable. The maximum optical polarization has been found to be associated with both the beaming factor (Fan et al. 1997) and the largest optical variation (Fan \& Feng 1998). Infrared polarization has been reported to be correlated with infrared spectral indices (Impey et al. 1982). Wills et al. (1992) found that polarization is associated with the core-dominance parameter. So, the extreme property of polarization in blazars could be explained as the effect of the relativistic model.

BL Lacertae $(2200+420)$, the archetype of its class, lies in a giant elliptical galaxy at a redshift of $\sim 0.07$ (Oke \& Gunn 1974; Thuan et al. 1975). It was thought to be a "variable star" by Schmit (1968) and was identified with the unusual radio source VRO 42.22 .01 by MacLeod \& Andrew (1968). The detection of variability in the radio flux (Biraud \& Veron 1968; Gower 1969) and the featureless spectrum (Oke et al. 1969; DuPuy et al. 1969) resulted in the suggestion that it is a BL Lac object (Strittmatter et al. 1972). Its early properties were reviewed by Stein et al. (1976) and Miller (1978). It is one of the best-studied objects in the optical and radio bands. Superluminal radio components have been observed (Vermeulen \& Cohen 1994; Fan et al. 1996). The optical and radio emissions are both variable and polarized, and the radio and optical polarizations are correlated (Sitko et al. 1985). The maximum polarizations obtained in the radio, infrared and optical bands are $P_{\text {Rad }}=10.0 \%$ (Gabuzda et al. 1989), $P_{\mathrm{IR}}=15.1 \%$ (Impey et al. 1984), and $P_{\text {Opt. }}=23 \%$ (Angel \& Stockman 1980) respectively. Recently, observations taken with EGRET on the Compton Gamma Ray Observatory (CGRO) between 1995 January 24 and 1995 February 14 indicate a flux of $(40 \pm 12) 10^{-8}$ photon $/ \mathrm{cm}^{2} / \mathrm{s}$ above $100 \mathrm{MeV}$ (Catanese et al. 1997), but there is no evidence of gamma rays in Whipple observations (Kerrick et al. 1995; Quinn et al. 1995). BL Lac has been observed in the optical bands for about 100 years (Fan et al. 1998). Webb et al. (1988) constructed its historic optical light curve. Very recently we 
Table 1. Literature of the near-infrared data for BL Lacertae

\begin{tabular}{|c|c|c|}
\hline Observer(s) & Points & Telescope(s) \\
\hline Epstein et al. (1972) & 3 & 100-inch \\
\hline Knacke et al. (1976) & 2 & PKBT $2.1 \mathrm{~m}$ \\
\hline O'Dell et al. (1977) & 1 & $\mathrm{UM} / \mathrm{UCSD} 1.5 \mathrm{~m}, 9^{\prime \prime}$ \\
\hline O'Dell et al. (1978) & 8 & $\mathrm{UM} / \mathrm{UCSD} 1.5 \mathrm{~m}, 9^{\prime \prime}, 18^{\prime \prime}$ \\
\hline Puschell \& Stein (1980) & 7 & $\mathrm{UM} / \mathrm{UCSD} 1.5 \mathrm{~m}, 18^{\prime \prime}$ \\
\hline Allen et al. (1982) & 1 & UKIRT $3.8 \mathrm{~m}$ \\
\hline Moore et al. (1982) & 2 & $\mathrm{UAO} 1.5 \mathrm{~m}$ \\
\hline Impey (1983) & 1 & Hawaii $2.2 \mathrm{~m}$, \\
\hline Sitko et al. (1983) & 5 & $\mathrm{UM} / \mathrm{UCSD} 1.5 \mathrm{~m}, 9^{\prime \prime}, 18^{\prime \prime}$ \\
\hline Puschell et al. (1983) & 1 & $\mathrm{UM} / \mathrm{UCSD} 1.5 \mathrm{~m}$ \\
\hline Impey et al. (1984) & 4 & UKIRT $3.8 \mathrm{~m}, 10^{\prime \prime}$ \\
\hline Holmes et al. (1984) & 2 & UKIRT $3.8 \mathrm{~m}, 10^{\prime \prime}$ \\
\hline Gear et al. (1985) & 3 & UKIRT \\
\hline Smith et al. (1987) & 11 & KNPO $2.1 \mathrm{~m}$ \\
\hline Brown et al. (1989) & 2 & UKIRT $3.8 \mathrm{~m}$ \\
\hline Bregman et al. (1990) & 6 & UKIRT $3.8 \mathrm{~m}$; Hale $5.0 \mathrm{~m}$ \\
\hline Mead et al. (1990) & 11 & UKIRT $3.8 \mathrm{~m}$ \\
\hline Kawai et al. (1991) & 3 & TCS $1.5 \mathrm{~m}$ \\
\hline Sitko \& Sitko (1991) & 15 & $\begin{array}{c}\text { KNPO } 1.3 \mathrm{~m} \& 1.5 \mathrm{~m} \\
6^{\prime \prime}, 11^{\prime \prime}, 12^{\prime \prime}, 23^{\prime \prime}\end{array}$ \\
\hline Takalo et al. (1992) & 4 & TCS $1.5 \mathrm{~m}, 15^{\prime \prime}$ \\
\hline Gear (1993) & 1 & UKIRT, $20^{\prime \prime}$ \\
\hline Lichfield et al. (1994) & 10 & ESO $2.2 \mathrm{~m}, 12^{\prime \prime}$ \\
\hline Xie et al. (1994) & 1 & $\mathrm{BAO} 1.26 \mathrm{~m}$ \\
\hline
\end{tabular}

have discussed its periodicity and found a 14-year period from the $B$ band light curve (Fan et al. 1998). BL Lacertae has been observed in the infrared since the beginning of the 1970s, but no long-term infrared properties have been discussed in the literature. In this paper, we will mainly study the variability and discuss the properties in the infrared. The paper has been arranged as follows: in Sect. 2, we give the bibliography of the data; in Sect. 3, we discuss them.

\section{Infrared variability}

\subsection{Data}

BL Lacertae has been observed in the near-infrared bands $(J, H$, and $K)$ for about 20 years. The data from the literature listed in Table 1 and the $38 K$ band data derived from the paper of Soifer \& Neugebauer (1980) are discussed in this paper. Table 1 gives the observer(s) in Col. 1; the number of data points in Col. 2, and the telescope(s) used in Col. 3.

\subsection{Variations}

BL Lacertae is located at $b=-10^{\circ}$ and thus has a reddening due to our own galaxy. According to the model

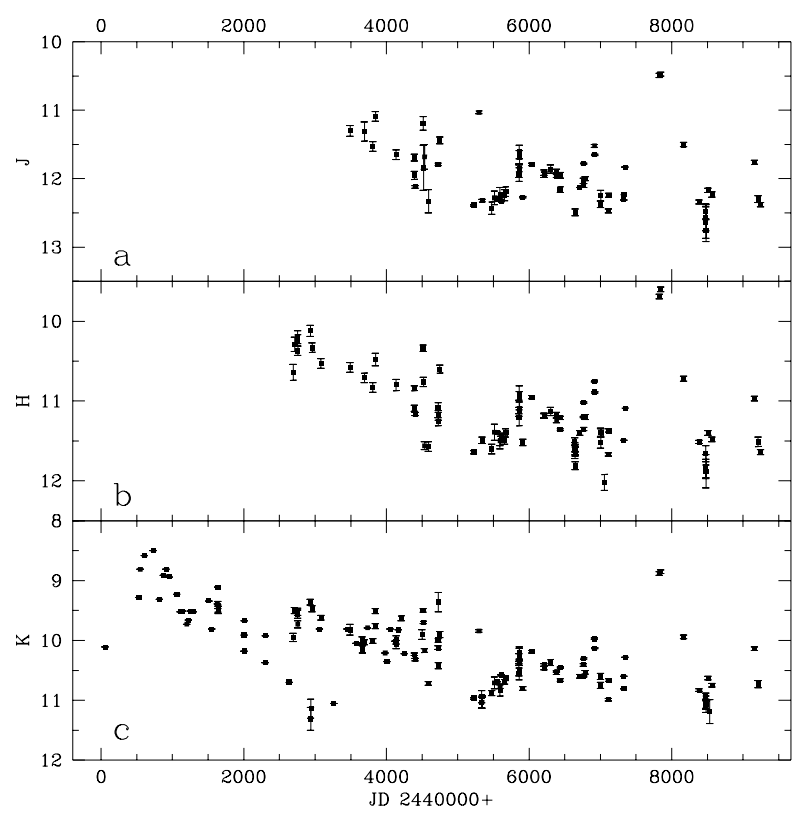

Fig. 1. a) The long-term $J$ light curve of BL Lacertae, b) The long-term $\mathrm{H}$ light curve, c) The long-term K light curve, some early data are derived from the paper of Soifer \& Neugebauer (1980)

of Sandage (1972), $A_{V}=0.165(1.192-\tan b) \csc b$, for $|b| \leq 50^{\circ}$, we have $A_{V}=0.97$. Following the reddening curve (Cruz-Gonzalez \& Huchra 1984, see also Whitford 1958): $A(\lambda)=A_{V}\left(0.11 \lambda^{-1}+0.65 \lambda^{-3}-0.35 \lambda^{-4}\right.$, we can get $A_{J}=0.267 \mathrm{mag}, A_{H}=0.158 \mathrm{mag}$, and $A_{K}=0.092 \mathrm{mag}$. After the correction, we got the infrared $(J, H$, and $K$ bands) light curves and show them in Fig. 1. The largest infrared amplitude of variability in the $J, H$, and $K$ bands: $\Delta J=2^{\mathrm{m}} 29(10.47-12.76), \Delta H=2^{\mathrm{m}} 42(9.60-12.02)$, $\Delta K=2 .{ }^{\mathrm{m}} 93(8.47-11.30)$ have been obtained from the available data. There is no correlation between color index and brightness; although there is some tendency of $J-H$ increasing with $J$, it is far from being conclusive (see Figs. 2a-c).

For color indices, we have got strong correlations of $J-H$ vs. $J-K$ and $J-K$ vs. $H-K: J-K=$ $(1.15 \pm 0.02)(J-H)+0.68 \pm 0.01$ with a Spearman Rank Correlation coefficient of $r=0.702$ and a probability of the correlation having occurred by chance $p=1.310^{-8}$; $H-K=(0.57 \pm 0.003)(J-K)-0.12 \pm 0.01$ with $r=0.796$ and $p=1.410^{-12}$, but no correlation was found for $J-H$ vs. $H-K$ (see Figs. 2d-f). We also found that the average values of color indices are $J-H=0.78 \pm 0.11$ ( 65 data),$H-K=0.78 \pm 0.12$ (71 data), and $J-K=1.55 \pm 0.13$ (63 data). One set of data $J=11.56, H=11.72$, and $K=10.26$ (Sitko et al. 1983) are not included in Fig. 2 because the color index of $J-H=0.23$ is much lower than the average value. If we only consider the data with known apertures 

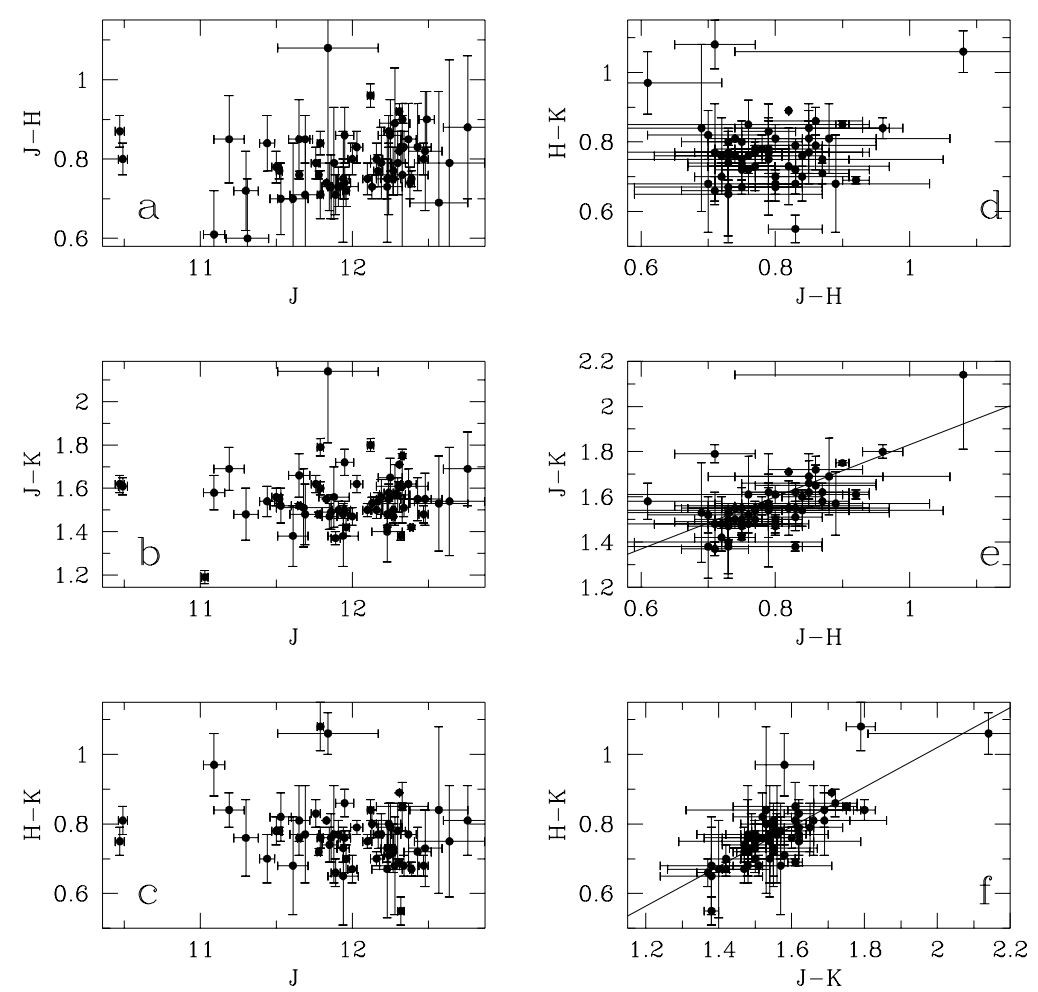

Fig. 2. a) Plot of $J$ against $J-H$; b) Plot of $J$ against $H-K$; c) Plot of $J$ against $J-K$; d) Color-Color Plot of $J-H$ vs. $H-K$; e) Color-Color Plot of $J-H$ vs. $J-K$. The straight line is for the best fit line; f) Color-Color Plot of $J-K$ vs. $H-K$. The straight line is for the best fit line

and make the aperture correction according to the method of Sandage (1972), there is no correlation between color index and magnitude or between $J-H$ and $H-K$ either.

\section{Discussion}

The largest observed amplitude of variability in the infrared bands are $\Delta J=2 \cdot 29, \Delta H=2 \cdot{ }^{\mathrm{m}} 42$, and $\Delta K=$ $2^{\mathrm{m}}$ 93. During the same period the largest observed amplitude of variability in the optical bands are: $\Delta U=$ $3^{\mathrm{m}} \cdot 39(14.58-17.97), \Delta B=3^{\mathrm{m}} \cdot 70(14.29-17.99), \Delta V=$ $3^{\mathrm{m}} \cdot 11(13.62-16.73), \Delta R=2^{\mathrm{m}} \cdot 73(13.26-15.99), \Delta I=$ $2^{\mathrm{m}} 54(12.56-15.10)$, which are slightly greater than that in the infrared. That result could be due to a larger contamination in the near-infrared by the underlying galaxy or to a better sampling of the light curve in the optical band. BL Lac is rapidly variable in the optical: by a factor of 2.5 over a time scale of 26 hours (Veron 1978), 1.0 over about one hour (Weistrop \& Goldsmith 1973), 0.5 over a few minutes (Weistrop 1973), daily variation as great as 0.3 (Caswell et al. 1974), and $0^{\mathrm{m}} 56$ over a short time scale of 40 minutes in the $B$ band (Xie et al. 1988); intraday variability of 0.21 (Heidt \& Wagner 1995), a variability by a factor of 2.6 over a time scale of 24 hours was observed in the $K$ band (Impey et al. 1984). Rapid variation in the infrared flux favors a nonthermal mechanism over thermal emission by dust (Cruz-Gonzalez \& Huchra 1984). For 3C 66A, De Diego et al. (1997) found that if the largest flux in the infrared is due to thermal processes, then the peak flux suggests that the emitting region is close to light months in diameter while the observed time scales of variability is much shorter. So violent variations in the infrared of BL Lacertae mean that the IR radiation cannot be thermal. In addition, high infrared polarization $P_{\mathrm{IR}}=15.1 \%$ reported in the $H$ band (Impey et al. 1984) suggests the infrared emission is non-thermal.

As for the spectrum and the brightness of the object, although there is a correlation between $B-I$ and $B: B=$ $(1.31 \pm 0.02)(B-I)+(13.03 \pm 0.1)$ with a correlation coefficient $r=0.67$ (Fan et al. 1998), there is almost no similar correlation in the infrared, which is probably due to the effect of the underlying galaxy diluting this kind of correlation.

There is a strong correlation between $J-H$ vs. $J-K$ and $J-K$ vs. $H-K$, but there is almost no correlation between $J-H$ and $H-K: J-K=(1.15 \pm 0.02)(J-H)+$ $0.70 \pm 0.02, H-K=(0.57 \pm 0.003)(J-K)-0.16 \pm$ 0.01 . Other radio selected BL Lac objects $(0219+428$, $0422+004,0735+178,0829+046$ and $1418+546$ ) (Massaro 
et al. 1995) show similar properties. They show strong color-color correlations both for $J-H$ and $J-K$ and for $J-K$ and $H-K$, but not for $J-H$ and $H-K$. We think that one of the reasons is probably due to the fact that $J-K$ has a wider distribution than $J-H$ and $H-K$. That $J-H$ and $H-K$ concentrate in a small region dilutes the correlation; another reason probably comes from the fact that the spectrum deviates from the power-law.

The long-term (about 20 years) infrared light curves have been presented, there are strong correlations between $J-K$ and $J-H$ as well as $H-K$. The infrared emission is nonthermal.

Acknowledgements. This work is supported by the National Pandeng Project of China.

\section{References}

Allen D.A, Ward M.J., Hyland H.R., 1982, MNRAS 199, 969

Angel J.R.P., Stockman H.S., 1980, ARA\&A 8, 321

Bailey J., Hough J.H., Axon D.J., 1983, MNRAS 203, 339

Biraud F., Véron P., 1968, Nat 219, 254

Bregman J.N., Glassgold A.E., Huggins P.J., et al., 1990, ApJ 352,574

Brown L.M.J., Robson E.I., Gear W.K., Smith M.G., 1989, ApJ 340, 150

Carswell R.F., Strittmatter P.A., Williams S.R., et al., 1974, ApJ 190, L101

Catanese M., Akerlof C.W., Williams R.E., et al., 1997, ApJ 480,562

Cruz-Gonzalez I., Huchra J.P., 1984, AJ 89, 441

De Diego J.A., Kidger M.R., Gonzales-Perez J.N., Lehto H.J., 1997, A\&A 318, 331

DuPuy D., Schmitt J., McClure R., van den Bergh S., Racine R., 1969, ApJ 156, L135

Epstein E.E., Fogarty W.G., Hachney K.R., et al., 1972, ApJ 178, L51

Fan J.H., Feng X.C., 1998, Publ. of Yunnan Observatory (in press)

Fan J.H., Xie G.Z., Pecontal E., et al., 1998, ApJ (accepted)

Fan J.H., Cheng K.S., Zhang L., Liu C.H., 1997, A\&A 327, 947

Fan J.H., Xie G.Z., Wen S.L., 1996, A\&AS 116, 409

Fan J.H., 1993, Ph.D. Thesis, Yunnan Astronomic Observatory, Chinese Academy of Sciences

Gabuzda D.C., 1989, ApJ 347, 701

Gear W.K., 1993, MNRAS 264, 919

Gear W.K., Robson E.I., Ade P.A.R., et al., 1985, ApJ 291, 511

George I.M., Warwick R.S., Bromage G.E., 1988, MNRAS 232, 793

Gower J.F.R., 1969, Nat 224, 569

Heidt J., Wagner S.J., 1995, A\&A 305, 42

Holmes P.A., Brand P.W.J.L., Wolstencroft R.D., Williams, P.M., 1984, MNRAS 210, 961

Impey C.D., Brand P.W.J.L., Wolstencroft R.D., Williams P.M., 1982, MNRAS 200, 19

Impey C.D., 1983, MNRAS 202, 397

Impey C.D., Brand P.W.J.L., Wolstencroft R.D., Williams P.M., 1984, MNRAS 209, 245
Kawai N., Matsuoka M., Bregman J.N., et al., 1991, ApJ 382, 508

Kerrick A.D., Akerlof C.W., Biller S., et al., 1995, ApJ 452, 588

Knacke R.F., Capps R.W., Johns M., 1976, ApJ 210, L69

Litchfield S.L., Robson E.I., Stevens J.A., 1994, MNRAS 270, 341

MacLeod J.M., Andrew B.H., 1968, ApL 1, 243

Massaro E., Nesci R., Perda G.C., et al., 1995, A\&A 299, 339

Mead A.R.G., Ballard K.R., Brand P.W.J.L., et al., 1990, A\&AS 83, 183

Miller J.S., 1978, Comm. Astrophys. 7, 175

Moore R.L., McGraw J.T., Angel J.R.P., et al., 1982, ApJ 260, 415

Morini M., Chiappetti L., Maccagni D., et al., 1986, ApJ 306, L71

O'Dell S.L., Pushell J.J., Stein W.A., 1977, ApJ 213, 351

O'Dell S.L., Pushell J.J., Stein W.A., Warner J.W., 1978a, ApJS 38, 267

Oke J.B., Gunn J.E., 1974, ApJ 189, L5

Oke J.B., Neugebauer G., Becklin, 1969, ApJ 156, L41

Pica A.J., Pollock J., Smith A.G., et al., 1980, AJ 85, 1442

Puschell J.J., Jones T.W., Pillips A., et al., 1983, ApJ 265, 625

Quinn J., et al., 1995, in Prof. 24th Int. Cosmic Ray Conf. (Rome) 2, 369

Sandage A., 1972, ApJ 178, 1

Schramm K.J., Borgeest U., Kuhl D., et al., 1994, A\&AS 106, 349

Sillanpaa A., Mikkola S., Valtaoja L., 1991, A\&AS 88, 225

Sitko M.L., Sitko A.K., 1991, PASP 103, 160

Sitko M.L., Schmidt G.D., Stein W.A., 1985, ApJS 59, 323

Sitko M.L., Stein W.A., Zhang Y.X., Wisniewski W.Z., 1983, PASP 95, 724

Smith P.S., Balonek T., Elston R., Heckert P.A., 1987, ApJS 64, 459

Soifer B.T., Neugebauer G., 1980, IAU Symps. 90, 329

Stein W.A., O'Dell S.L., Strittmatter P.A., 1976, ARA\&A 14, 173

Strittmatter P.A., Carswell R.F., Stein W.A., et al., 1972, ApJ 175, L7

Takalo L.O., Sillanpaa A., Nilsson K., et al., 1992, A\&AS 94, 37

Takalo L.O., Sillanpaa A., Pursimo T., et al., 1996, A\&AS 120, 313

Thompson D.J., Bertsch D.L., Dingus B.L., et al., 1995, ApJS 101,259

Thompson D.J., Bertsch D.L., Dingus B.L., et al., 1996, ApJS 107,227

Thuan T.X., Oke J.B., Gunn J.E., 1975, ApJ 201, 45

Tornikoski M., Valtaoja E., Terasranta H., et al., 1994, A\&A 289,673

Vermeulen R.C., Cohen M.H., 1994, ApJ 430, 467

Urry C.M., 1984, Ph.D. Thesis, the Univ. of Hopkins

Véron-Cetty M.P., Véron P., 1996, ESO Sci. Report, No. 17

Véron P., 1978, in Pittb. Conf. on BL Lac Objects, Wolf A. (ed.), p. 20

Webb J.R., Smith A.G., Leacock R.J., et al., 1988, AJ 95, 374

Weistrop D., 1973, Nat 241, 157

Weistrop D., Goldsmith S., 1973, ApL 14, 225

Whitford A.E., 1958, ApJ 107, 102

Wills B.J., Wills D., Breger M., et al., 1992, ApJ 398, 454

Xie G.Z., Lu R.W., Zhou Y., et al., 1988, A\&AS 72, 163

Xie G.Z., Li K.H., Zhang Y.H., et al., 1994, A\&AS 106, 361 